Original

\title{
Resultados a medio plazo del tratamiento endourológico retrógrado con balón de la estenosis pieloureteral en niños menores de 1 año
}

\author{
Alberto Parente Hernández, José $\mathrm{M}^{\mathrm{a}}$ Angulo Madero, Rosa $\mathrm{M}^{\mathrm{a}}$ Romero Ruiz, Susana Rivas Vila, \\ Ana Laín Fernández, María Fanjul Gómez \\ Sección de Urología Pediátrica, Servicio Cirugía Pediátrica. Hospital Infantil Gregorio Marañón. Madrid, España
}

\section{Resumen}

Introducción: En los últimos años se han extendido terapéuticas mínimamente invasivas para el tratamiento de la estenosis pieloureteral en niños. Algunas de ellas con pobres resultados en niños menores de 4 años. Presentamos nuestra experiencia en el tratamiento mediante dilatación endourológica retrógrada con balón de alta presión en lactantes.

Material y métodos: Presentamos un estudio retrospectivo de 16 pacientes menores de 1 año tratados en nuestra unidad. El seguimiento medio tras la intervención es de 27,4 $\pm 10,0$ meses. El protocolo diagnóstico incluyó ecografía abdominal, gammagrafỉa renal, cistouretrografía miccional y renograma diurético. El tratamiento se realizó mediante dilatación endoscópica retrógrada bajo control radioscópico. Los balones utilizados eran en todos los casos semicompliantes, con un perfil de $5 \mathrm{~mm}, 6 \mathrm{~mm}$ ó $7 \mathrm{~mm}$. Tras la dilatación se colocó stents tipo doble $\mathrm{J}$ de calibre y longitud en función del peso del paciente. Para obtener los resultados se analizan las ecografías y los renogramas diuréticos por lo menos 1 año después de la intervención.

Resultados: La duración media de la intervención fue de 36,4 $\pm 15,5$ minutos. Durante la intervención, en 2 pacientes no fue posible la colocación del stent, El tiempo de ingreso fue de 48 horas en todos los pacientes excepto en 1 de ellos. Las necesidades analgésicas fueron exclusivamente AINES. El stent tipo doble $\mathrm{J}$ fue retirado mediante cistoscopia de manera ambulatoria. En 3 pacientes hubo complicaciones postoperatorias: una migración del stent, una ITU y un urinoma. La técnica fracasó en 1 paciente que necesitó de pieloplastia desmembrada. En los controles se observó la desaparición de la hidronefrosis en todos los casos, desapareciendo así mismo la dilatación de los cálices renales. El renograma diurético MAG-3 mejoró en todos los lactantes, siendo el patrón de la curva normal en 14 casos y semiobstructivo en 2 . El tiempo medio de eliminación fue de 9,8 92,8 minutos.

Conclusiones: Pensamos que la dilatación con balón es una técnica mínimamente agresiva que es posible realizar en pacientes menores de 1 año con buenos resultados y escasas complicaciones.

Palabras clave: Lactante. stenosis pieloureteral, dilatación, balón, endourológica.

\section{Medium-term results of the endourological management with balloon dilatation of the ureteropelvic junction stenosis in infants}

\begin{abstract}
Aim: In the last years it has spread minimally invasive therapeutic for the treatment of pyeloureteral junction obstruction in children. Some of them have got poor outcomes in 4-year-old minor children. We show our experience in the retrograde dilatation with balloon of high pressure in infants.

Methods: It's a retrospective study of 16 infants treated in our hospital. The average follow-up after the intervention is $27.4 \pm 10.0$ months. The diagnostic protocol included abdominal ultrasound, cystogram and diuretic renography. The treatment was realized by endourology retrograde balloon dilatation under fluoroscopy. The balloons were in all the cases semicompliant, with a profile of $5 \mathrm{~mm}, 6 \mathrm{~mm}$ ó $7 \mathrm{~mm}$. After the expansion there was placed stents type double $\mathrm{J}$ with profile and length depending on the weight of the patient. To obtain the results, we do ultrasound scans and diuretic renography at least 1 year after the intervention.

Results: The mean duration of the intervention was 36.4 \pm 15.5 minutes. During the intervention, in 2 patients the placement of the stent was not possible. The time of revenue was 48 hours in all the patients except in 1 of them. The analgesic needs were exclusively non steroid antiinflammatorys. The double $J$ stent was withdrawn with cystoscopy. In 3 patients there were postoperatory complications: a migration of the stent, a urinary infection and an urinoma. In 1 patient with worsening of hydronephrosis was needed of pyeloplasty. In the controls the disappearance of the hydronephrosis was observed in all the cases, eliminating likewise the expansion of the renal calyces. The diuretic renography improved in all babies, being the boss of the curve normal in 14 cases and semi obstructively in 2 . The mean time of elimination was $9.8 \pm 2.8$ minutes.

Conclusions: We think that the balloon dilatation of pyeloureteral junction obstruction is a minimally aggressive technique that is possible to realize in infants with good results and scanty complications.
\end{abstract}

Keywords: Infant, pyeloureteral junction obstruction, dilatation, balloon, endourology. 
T a pieloplastia desmembrada de Anderson-Hynes Lsigue siendo hoy día el "gold-standar" en el tratamiento de la estenosis pieloureteral en niños debido a que las distintas series muestran una tasa de curación cercana al 94\% ${ }^{1,2}$. Sin embargo, asocia una importante morbilidad que ha obligado a la búsqueda de otras técnicas mínimamente invasivas que disminuya los problemas creados por la cirugía sin disminuir la tasa de éxito de la intervención ${ }^{3}$. Así, han surgido otras alternativas, entre ellas técnicas endourológicas y laparoscópicas, la mayoría tras un periodo de adaptación en adultos. Dentro de éstas se ha descrito el uso en niños de la endopielotomía anterógrada y retrógrada con corte puro o mediante laser holmium, la dilatación con balón, la pieloplastia desmembrada laparoscópica, etc ${ }^{4-10}$. Así mismo, han sido descritas técnicas endourológicas en el tratamiento del megauréter obstructivo ${ }^{11}$.

La mayor parte de estas nuevas intervenciones tienen mayor dificultad técnica en niños de pequeño tamaño, lo que produce que muchas series muestren peores resultados en menores de 4 años $^{12}$. Sin embargo, los avances en ingeniería médica, especialmente al lograr instrumental endourológico de menor perfil pero que mantiene intactas cualidades como la alta presión de inflado en los balones de dilatación o la facilidad de uso de tutores y guías, han ayudado a mejorar estos resultados al facilitar las intervenciones endourológicas. Además, la mayor experiencia de los cirujanos implica un mejor aprovechamiento de los nuevos materiales para lograr un mayor porcentaje de éxito en la intervención. Así, hoy día es posible el uso de la mayoría de estas técnicas incluso en lactantes ${ }^{13}$. Presentamos en este trabajo nuestra experiencia en el tratamiento de la estenosis pieloureteral en menores de un año mediante dilatación endourológica retrógrada con balón de alta presión.

\section{MATERIAL Y MÉTODOS}

Hemos realizado un estudio retrospectivo de los pacientes menores de 1 año, del total de 54 niños tratados en nuestra unidad por estenosis pieloureteral entre julio de 2004 y diciembre de 2006. Así, se han analizado un total de 16 pacientes, 10 niños y 6 niñas, con EPU congénita que requirieron tratamiento quirúrgico antes del año de vida. Se han excluido del estudio aquellos pacientes que presentaban otras patologías asociadas (megaureter obstructivo del lado contralateral, etc). En todos los casos el tratamiento se realizó mediante dilatación endourológica retrógrada con balón de alta presión. La edad media en el momento de la dilatación fue de $7,93 \pm 2,14$ meses. El peso medio de los pacientes fue $6,230 \pm 2,542 \mathrm{~kg}$, siendo el paciente de menor peso de $3.430 \mathrm{~kg}$. El seguimiento medio tras la intervención en el momento de realizar el estudio es de $27,4 \pm 10,0$ meses.

En 9 casos la hidronefrosis era derecha y en 7 casos izquierda. Hasta 12 de los casos (73.3\%) tenían diagnóstico prenatal de ectasia piélica, que fue confirmada mediante ecografía antes del $7^{\circ}$ día de vida. En 2 de los casos el diagnóstico se realiza tras infección del tracto urinario (ITU), por lo que se realiza estudio de la vía urinaria siguiendo el protocolo de estudio de ITU en menores de 3 meses, en 1 caso por sospecha de masa abdominal en la exploración abdominal y en otro caso como hallazgo casual durante una exploración ecográfica. Hasta 6 de los pacientes $(40 \%)$ padecieron ITUs antes del tratamiento quirúrgico.

El protocolo diagnóstico incluyó ecografía abdominal, gammagrafía renal con DMSA en aquellos pacientes con diagnóstico prenatal, cistouretrografía miccional seriada (CUMS) y renograma diurético MAG-3 (mercapto-acetil-glicina III). En la ecografía abdominal los diámetros anteroposteriores de la pelvis renal se encontraban aumentados en todos los casos, con una media de 23,1 $\pm 6,1 \mathrm{~mm}$. Asimismo, en todos los casos se observó dilatación de los cálices renales con adelgazamiento de la cortical renal, siendo este mayor o menor en función del caso. La CUMS fue normal en todos los pacientes. El renograma diurético mostró un patrón obstructivo patológico de la curva con un tiempo medio de eliminación del contraste muy superior a 30 minutos en todos los lactantes. La función renal se encontraba disminuida por debajo del $40 \%$ en 4 pacientes, mostrando una hiperfunción compensadora o paradójica 7 lactantes. En los otros 5 casos, en los que se encontraba conservada la función renal, la ecografía renal mostró un aumento progresivo de la dilatación de la pelvis renal.

El tratamiento se realizó mediante dilatación endoscópica retrógrada bajo anestesia general con control radioscópico. Para ello se realizó cistoscopia (Storz 10), localizándose ambos meatos ureterales. Se logró tutorizar el uréter del lado afecto con catéter ureteral de $4 \mathrm{Fr}$ excepto en 3 casos en los que se tuvo que utilizar tutores de $3 \mathrm{Fr}$ por el pequeño 
tamaño del meato. En todos los casos se realizó pielografía retrógrada para objetivar la dificultad de eliminación del contraste por el riñón y contrastar la pelvis renal, siendo este proceso necesario para lograr visualizar la localización de la unión pieloureteral. Se colocaron guías ureterales de 0,014“ hasta pelvis renal a través del catéter ureteral (PT2, Boston Scientific), a través del cual se logró ascender el balón de dilatación. Los balones utilizados eran en todos los casos semicompliantes (Terumo®), con un perfil de inflado a presión de 12 atm de 5 $\mathrm{mm}$ en 11 pacientes, $6 \mathrm{~mm}$ en 2 pacientes y $7 \mathrm{~mm}$ en los otros 3. La dilatación fue controlada mediante radioscopia y control de la presión de inflado. Se consideró dilatada la unión pieloureteral al desaparecer la muesca del balón, extravasándose contraste en 5 casos (Fig. 1). Tras la dilatación se colocó stents tipo doble $J$ sobre la misma guía $\left(0,014^{\prime \prime}\right)$ de calibre y longitud en función del peso del paciente. En 5 casos este fue de 3 Fr y $12 \mathrm{~cm}$, en 8 casos de 4 Fr y $12 \mathrm{~cm}$, y en 1 caso de 4.8 Fr y $14 \mathrm{~cm}$. En 2 pacientes no fue posible su colocación por dificultades técnicas (Tabla 1).

$\mathrm{El}$ stent tipo doble $\mathrm{J}$ fue retirado mediante cistoscopia durante el cual se realizó un nuevo calibrado de la unión pieloureteral. Este procedimiento se realizó de manera ambulatoria, a una media de 45,5 \pm 12,6 días después de su colocación. En un

\section{Tabla 1}

\begin{tabular}{rcccc}
\hline Paciente & $\begin{array}{c}\text { Tutor } \\
\text { (Fr) }\end{array}$ & $\begin{array}{c}\text { Balón } \\
\text { (mm) }\end{array}$ & $\begin{array}{c}\text { Longitud } \\
\text { doble J } \\
\text { (cm) }\end{array}$ & $\begin{array}{c}\text { Calibre } \\
\text { doble J } \\
\text { (Fr) }\end{array}$ \\
\hline 1 & 4 & 5 & 12 & 4 \\
2 & 4 & 5 & 12 & 4 \\
3 & 4 & 5 & 12 & 3 \\
4 & 4 & 5 & 12 & 3 \\
5 & 4 & 5 & 12 & 3 \\
6 & 3 & 5 & 12 & 3 \\
7 & 3 & 7 & 14 & 4,8 \\
8 & 4 & 5 & 14 & 4 \\
9 & 4 & 7 & 14 & 4 \\
10 & 4 & 6 & 12 & 3 \\
11 & 4 & 5 & 12 & 4 \\
12 & 4 & 6 & 14 & 4 \\
13 & 3 & 7 & - & - \\
14 & 4 & 5 & 16 & 4 \\
15 & 4 & 5 & 12 & 4 \\
16 & 3 & 5 & - & - \\
\hline
\end{tabular}
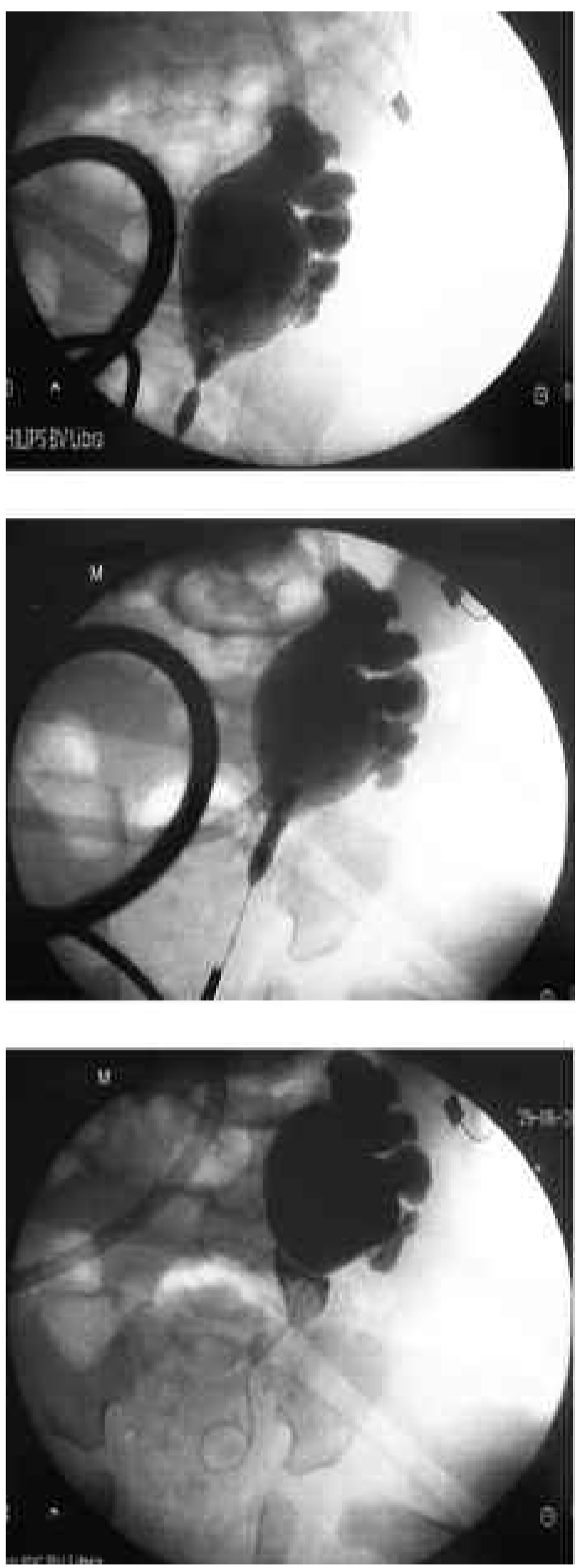

FIGURA 1. Dilatación de EPU con extravasación de contraste y colocación de doble $J$ 
caso fue necesaria la colocación de un nuevo stent al observarse muesca en el balón utilizado para la calibración. Para facilitar su retirada se unió el stent a una sutura que se exteriorizó a través de la uretra, por lo que pudo ser retirada sin incidencias en la consulta a las 3 semanas de la nueva dilatación (Fig. 2).
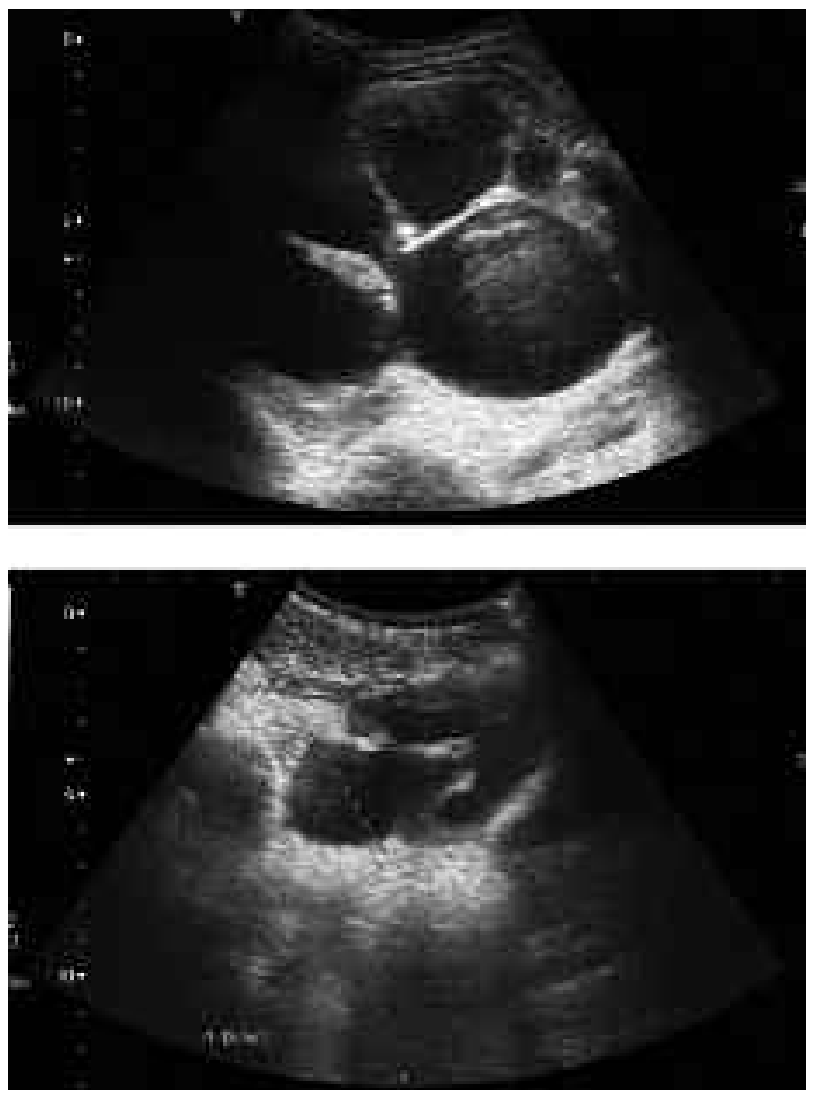

FIGURA 2. Ecografia preoperatoria y tras 1 año postoperatorio, observándose la disminución progresiva de la dilatación.

Se ha estudiado el tiempo de ingreso hospitalario, las necesidades analgésicas de los lactantes, las complicaciones intraoperatorias y postoperatorias así como la necesidad de reintervención. Para analizar los resultados de la técnica se han analizado las ecografías y los renogramas diuréticos en todos los pacientes por lo menos 1 año después de la intervención, no siendo incluidos en el estudio aquellos pacientes que no tienen estudios de seguimiento completos tras un año de la dilatación.

\section{RESULTADOS}

La duración media de la cirugía fue de 36,4 15,5 minutos. Durante la intervención, sólo en un paciente no fue posible la colocación del stent, desarrollándose el resto de intervenciones sin incidencias. En este paciente se realizaron controles ecográficos seriados durante el postoperatorio, mostrando una disminución progresiva de la dilatación sin otras complicaciones. El tiempo de ingreso fue de 48 horas en todos los pacientes excepto en 1 de ellos que permaneció 72 horas por hematuria. Durante su ingreso las necesidades analgésicas fueron exclusivamente AINES, añadiéndose en 2 de ellos un catéter caudal para analgesia locorregional, aumentando de este modo la confortabilidad del lactante durante su estancia hospitalaria.

En 4 pacientes hubo complicaciones postoperatorias: una migración del stent tipo doble $\mathrm{J}$ hacia pelvis renal que fue retirado mediante ureteroscopia; una ITU que obligó a una retirada precoz del stent; un urinoma que necesitó la colocación de una nefrostomía percutánea durante 2 semanas, siendo retirada tras la realización de un nefrostograma que mostraba un buen paso del contraste a través de unión pieloureteral. En los 3 casos las complicaciones fueron resueltas sin incidencias, siendo su evolución a medio plazo buena con resolución del patrón obstructivo en el renograma diurético realizado al año de la dilatación. El $4^{\circ}$ paciente, en el cual no fue posible la colocación del stent por problemas técnicos, requirió de nefrostomía percutánea por aumento agudo de la hidronefrosis tras la dilatación. En este paciente fue necesario realizar una pieloplastia desmembrada según técnica convencional tras comprobar la ausencia de paso del contraste en el nefrostograma realizado posteriormente, siendo su evolución posterior satisfactoria. Así, dos pacientes han necesitado hasta el momento de reintervención tras un periodo medio de seguimiento de $27,4 \pm 10,0$ meses

$\mathrm{Al}$ analizar los controles ecográficos y renográficos posteriores a la dilatación, realizados todos ellos al menos 1 año después de la intervención (incluido el paciente que requirió de pieloplastia desmembrada), encontramos que el diámetro medio de la pelvis renal fue de 7,0 $\pm 4,4 \mathrm{~mm}$, siendo las diferencias pre-postdilatación estadísticamente significativas (p<0.001) (Fig. 3). La disminución de la hidronefrosis se produjo en todos los casos, desapareciendo así mismo la dilatación de los cálices renales. El renograma diurético MAG-3 mejoró en todos los lactantes. El patrón de la curva fue normal en 14 casos y semiobstructivo en 2, teniendo estos pacientes 


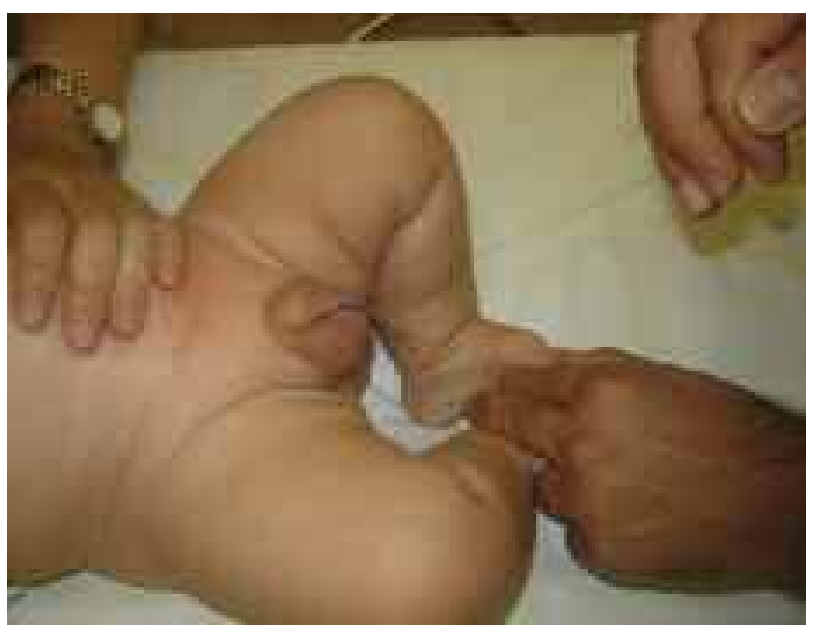

FIGURA 3. Retirada de doble J unido a sutura en la consulta.

una gran mejoría ecográfica de la hidronefrosis con diámetros de la pelvis renal de 4 y $8 \mathrm{~mm}$. El tiempo medio de eliminación era de $9,8 \pm 2,8$ minutos, permaneciendo en todos los casos por debajo de 14 minutos. La función renal diferencial se normalizó en todos los pacientes con hiperfunción renal compensadora previa y mejoró muy levemente en aquellos que tenían pérdida de función renal previa a la dilatación. En aquellos pacientes que tenían función renal diferencial previa a la intervención normal, permaneció dentro de valores normales (Tabla 2).

\section{DISCUSIÓN}

Pese a que el tratamiento mediante dilatación retrógrada con balón de alta presión fue descrito inicialmente en adultos, diversos autores iniciaron su uso en niños. Así, se ha descrito la utilización del balón de Fogarty ${ }^{11}$, del Acucise ${ }^{12}$ o el uso del stent tipo pigtail hasta la llegada del balón de alta presión $^{11,13}$. Paralelamente otras técnicas endourológicas han sido descritas, como la endopielotomía incisional anterograda en $1986^{14}$ o retrógrada en $1986^{15}$. Sin embargo, este es el primer trabajo que analiza la utilidad de la dilatación en el tratamiento de la estenosis pieloureteral en menores de 1 año. Aunque algunos autores aseguran no encontrar indicación a los tratamientos endourológicos en niños de menos de 4 años ${ }^{9}$, nosotros pensamos, después de una larga experiencia en niños preadolescentes, que la no utilización del material adecuado para realizar la dilatación conlleva unos peores resultados respecto a la técnica clásica. La mejoría en los resultados en estos pacientes debe pasar por una mejor optimización del material a partir de la experiencia del cirujano. En nuestra experiencia si instrumentamos con tutores, balones y stents de calibre menor a 4 Fr. lograremos dilatar con éxito la unión pieloureteral, mientras que si lo hacemos por encima de $5 \mathrm{Fr}$. fracasaremos.

Nuestra serie muestra unos excelentes resultados a medio plazo con una tasa de curación completa del

Tabla 2

\begin{tabular}{|c|c|c|c|c|c|c|}
\hline $\mathbf{N}^{\mathbf{o}}$ & DAP pelvis pre & Fx renal pre & T 1/2 pre & DAP pelvis post & Fx renal post & T $1 / 2$ post \\
\hline 1 & 16 & 56 & $>30^{\circ}$ & 6 & 48 & $12^{-}$ \\
\hline 2 & 14 & 57 & $>30^{\circ}$ & 6 & 49 & $7^{-}$ \\
\hline 3 & 36 & 57 & $>30^{\circ}$ & 13 & 49 & $14^{-}$ \\
\hline 4 & 21 & 50 & $>30^{\circ}$ & 8 & 51 & $14^{-}$ \\
\hline 5 & 30 & 56 & $>30^{\circ}$ & 12 & 52 & $11^{\circ}$ \\
\hline 6 & 18 & 49 & $>30^{-}$ & 4 & 53 & $7^{-}$ \\
\hline 7 & 20 & 49 & $>30^{\circ}$ & 7 & 49 & $6^{-}$ \\
\hline 8 & 22 & 39 & $>30^{\circ}$ & 18 & 44 & $11^{\circ}$ \\
\hline 9 & 20 & 48 & $>30^{\circ}$ & 4 & 48 & $10^{-}$ \\
\hline 10 & 18 & 57 & $>30^{\circ}$ & 4 & 50 & $10^{-}$ \\
\hline 11 & 40 & 36 & $>30^{\circ}$ & 15 & 41 & $13^{-}$ \\
\hline 12 & 20 & 39 & $>30^{\circ}$ & 5 & 47 & $10^{-}$ \\
\hline 13 & 18 & 60 & $>30^{\circ}$ & 4 & 52 & $7^{-}$ \\
\hline 14 & 20 & 57 & $>30^{\circ}$ & 3 & 51 & $6^{-}$ \\
\hline 15 & 18 & 51 & $>30^{\circ}$ & 4 & 51 & $13^{-}$ \\
\hline 16 & 35 & 35 & $>30^{\circ}$ & - & - & - \\
\hline
\end{tabular}


$81,2 \%$, mejorando ostensiblemente el renograma diurético y la ecografía en otros 2 pacientes. Al no realizar en esta técnica reducción de la pelvis renal la mejoría en las pruebas de imagen no es inmediata, por lo que debemos considerar como válidos los controles a partir del año de la intervención. Algunos autores no consideran necesario la realización de renogramas en el seguimiento más que en casos seleccionados, siendo suficiente con controles ecográficos para observar la mejoría de la hidronefrosis $^{9}$. En este protocolo podríamos considerar nuestra tasa de éxito en el 93,7\%. Sin embargo, nuestra serie es pequeña por lo que no podemos pensar que se trata de una técnica exenta de defectos, sino que probablemente al aumentar nuestra serie encontraremos pacientes en los que no se logre la resolución de la estenosis pieloureteral. Algunos autores han demostrado que la mayoría de las recidivas suceden durante el primer año postoperatorio ${ }^{16,17}$, aunque un porcentaje de ellas pueden aparecer a medio o largo plazo ${ }^{18,19}$. Esto hace que estos pacientes deban seguir controles ecográficos por lo menos hasta la adolescencia para asegurar que la curación es completa. Así, nuestros resultados deben considerarse no definitivos al ser nuestro periodo de seguimiento postoperatorio de 27 meses y los pacientes menores de 1 año.

En un paciente no se logró realizar la dilatación con éxito, por lo que fue necesario realizar posteriormente una pieloplastia desmembrada. Tras la realización de este trabajo otro paciente requirió reintervención bajo la técnica clásica por fracaso en la dilatación. No encontramos mayor dificultad técnica durante la realización de la pieloplastia en ambos casos. Pensamos que una dilatación previa no empeora el pronóstico ni dilata el tiempo quirúrgico durante la intervención, a diferencia del resto de técnicas en las que el aumento de la fibrosis cicatricial en la unión pieloureteral dificulta el acceso a la zona quirúrgica. Como cirujanos pensamos que no debemos estar cerrados a una única técnica quirúrgica sino asumir las ventajas de cada una de ellas y poder modificar la indicación quirúrgica en función de las necesidades del paciente.

El tiempo operatorio medio y la estancia hospitalaria en nuestra serie, así como las necesidades analgésicas, se encuentran por debajo de la media habitual en una pieloplastia clásica. Así, podemos considerar la dilatación con balón de alta presión una técnica poco agresiva y que produce poco dolor en el lactante. Evitamos de esta manera las complicaciones habituales de la técnica abierta, al igual que el resto de técnicas endourológicas. Nuestras complicaciones son escasas, aunque dos de ellas requirieron de una nueva intervención: una migración del stent que obligó a realizar una ureteroscopia para su retirada y un urinoma que fue resuelto mediante la colocación de una nefrostomía percutánea. Aunque el resultado en ambos casos fue satisfactorio debemos considerar ambas complicaciones como graves. La extravasación de contraste tras la dilatación no debe ser considerada como complicación; ya algunos autores consideran necesaria la presencia de esta extravasación durante las endopielotomías para considerar que esta ha sido realizada con éxito ${ }^{20}$. No existe en nuestra serie ningún paciente que haya necesitado transfusión, a diferencias de las endopielotomías realizadas con laser Holmium o con corte clásico. Esto puede ser debido a que la dilatación rompe la estenosis sin cortar las estructuras que se puedan encontrar cerca, significando una importante ventaja respecto a otras técnicas endourológicas ${ }^{21,22}$. Además, la presencia de vaso polar como causa de la obstrucción en lactantes, a diferencia de adultos o de adolescentes, es improbable ${ }^{23}$, por lo que no consideramos necesario la realización de ninguna prueba de imagen previa a la intervención para descartar su presencia. Cuando dilatamos una estructura circular la cicatrización tiende a cerrarla de nuevo, por lo que pensamos que en dilataciones menores de $5 \mathrm{~mm}$ se reproducirá el defecto. El calibre que debemos intentar dar a la unión pieloureteral debe ser el adecuado para la edad y el tamaño del paciente. Así, el fracaso en la dilatación puede deberse a intentar minimizar la dilatación utilizando balones de calibres no adecuados al lactante, ya que no lograremos un calibre adecuado para el correcto vaciado de la pelvis renal. De igual manera el uso de guías hidrofílicas puede ayudarnos a vencer el paso en algunos pacientes complejos de la unión pieloureteral. En nuestro caso usamos stent tipo doble $\mathrm{J}$ de 3 ó $4 \mathrm{Fr}$, adecuados a la edad del paciente. Aunque su manejo puede resultar más sencillo a mayor diámetro del stent, no debemos intentar colocar un doble $J$ que fisiológicamente no sea adecuado para el tamaño del uréter.

Pensamos que la dilatación con balón de alta presión es una opción válida dentro de los tratamientos mínimamente invasivos de la estenosis pieloureteral. 
Probablemente otras opciones terapéuticas también mejoren sus resultados en los próximos años. Por el momento pensamos que la dilatación con balón es una técnica mínimamente invasiva que es posible realizar en lactantes con buenos resultados, escasas complicaciones $\mathrm{y}$, en caso de fracaso, recurrir a la técnica clásica sin dificultades técnicas añadidas.

\section{REFERENCIAS}

1. Persky L, McDougal WS, Kedia K: Management of inicial pyeloplasty failure. J Urol 1981;125(5):695-657.

2. Rohrmann D, Snyder HM III, Duckett JW Jr, Canning D, Zderic SA: The operative management of recurrent ureteropelvic junction obstruction. J Urol 1997;158(3 Pt 2):1257-1259.

3. Pardalidis NP, Papatsoris AG, Kosmaoglou EV: Endoscopic and laparoscopic treatment of ureteropelvic junction obstruction. J Urol 2002;168(5):1937-1940.

4. Moore RG, Avert TD, Schulman PG, Adams JB, Chen RN, Kavoussi LR: Laparoscopic pyeloplasty: experience with the initial 30 cases. J Urol 1997;157(2):459-462.

5. Janetschek G, Peschel R, Bartsch G: Laparoscopic Fenger plasty. J Endourol 2000;14(1):889-993.

6. Janetschek G, Peschel R, Frauscher F: Laparoscopic pyeloplasty. Urol Clin North Am. 2000;27(4):695-704.

7. Biyani CS, Cornford PA, Powell CS: Ureteroscopic endopyelotomy with the Holmium-YAG laser, mild-term results. Eur Urol 2000;38(2): 139-143.

8. Cohen TD, Gross MB, Preminger GM: Long-term follow-up of Acucise incision of ureteropelvic junction obstruction and ureteral strictures. Urology. 1996 Mar;47(3):317-323.

9. Rivas S, Romero R, Angulo JM, Sánchez-Paris O, Del Cañizo A, Parente A, Laín A, Fanjul M, Vazquez J: Effectiveness of high pressure balloon dilatation in the treatment of postsurgical strictures of urinary tract in children. Cir Pediatr. 2007;20(3): 183-187.

10. Parente A, Angulo JM, Romero R, Cañizo A, Laín A, Vázquez J: Tratamiento endourológico de la estenosis pieloureteral congénita. Actas UrolEsp2006;30(9):933-938.

11. Angulo JM, Arteaga R, Rodríguez Alarcón J, Calvo MJ: Role of retrograde endoscopic dilatation with balloon and derivation using double pig-tail catheter as an initial treatment for vesico-ureteral junction stenosis in children. Cir Pediatr. 1998;11 (1):15-18.

12. Braga LH, Lorenzo AJ, Skeldon S, Dave S, Bagli DJ, Khoury AE, Pippi Salle JL, Farhat WA: Failed pyeloplasty in children: Comparative analysis of retrograde endopyelotomy versus redo pyeloplasty. J Urol 2007;178(6):2571-2578.

13. Figenshau RS, Clayman RV: Endourologic options for management of ureteropelvic junction obstruction in the pediatric patient. Urol Clin North Am. 1998;25(2):199-209.

14. Doraiswamy NV: Retrograde ureteroplasty using ballon dilatation in children with pelviureteric obstruction. J Pediat Surg 1994:29(7);937-940.
15. Bolton DM, Bogaert GA, Mevorach RA, Kogan BA, Stoller ML: Pediatric ureteropelvic junction obstruction treated with retrograde endopyelotomy. Urology 1994;44(4):609-613.

16. Sugita Y, Clarnette TD, Hutson JM: Retrograde ballon dilatation for primary pelviureteric junction stenosis in chidren. $\mathrm{Br} \mathrm{J}$ Urol 1996;77(4):587-589.

17. Badlani G, Eshgi M, Smith AD: Percutaneus surgery for ureteropelvic junction obstruction: Technique and early results. 1986 135(1):26-28.

18. Inglis JA, Tolley DA: Ureteropelvic pyelolysis for pelviureteric junction obstruction. Br J Urol 1986;58:(3)250-252.

19. Jabbour ME, Golgfischer ER, Klima WJ, Stravodimos KG, Smith $\mathrm{AD}$ : Endopyelotomy after failed pyeloplasty: the longterm results. J Urol 1998 Se;160(3 Pt 1):690-692.

20. Kletscher BA, Segura JW, Le Roy AJ, et al: Percutaneous antegrade endopyelotomy: review of 50 consecutive cases. J Urol 1995 Mar; 153(3 Pt 1):701-703.

21. Di Marco DS, Gettman MT, Chow GK, LeRoy AJ, Segura JW: Long-term success of ureteropelvic junction obstruction repair comparing antegrade endopyelotomy to pyeloplasty. J Endourol 2005;58(Supplement 1), abstract MP33-02.

22. Knudsen BE, Cook AJ, Watterson JD, et al: Percutaneous antegrade endopyelotomy: long-term results from one institution. Urology 2004;63(2):230-234.

23. Desai MM, Desai MR, Gill AS: Endopyeloplasty versus endopyelotomy versus laparoscopic pyeloplasty for primary ureteropelvic junction obstruction. Urology2004;64(1):16-21;.

24. Minervini A, Davenport K, Keeley FX Jr, Timoney AG: Antegrade versus retrograde endopyelotomy for pelvic-ureteric junction obstruction. Eur Urol 2006;49(3):536-542.

25. Vaarala MH, Marttila T, Paananen I, Hellstrom P: Retrospective analysis of long-term outcomes of 64 patients treated by endopyelotomy in two low-volume hospitals: Good and durable results. J Endourol 2008(8);1659-1664.

26. Nakada SY, Wolf JS, Brink JA, Quillen SP, Nadler RB, Gaines $\mathrm{MV}$, et al: Retrospective analysis of the effect of crossing vessels on retrograde endopyelotomy outcomes using spiral CT angiography. J Urol 1998;159(1):62-65.

27. Turk IA, Davis JW, Winkelmann B, Deger S, Richter F, Fabrizio MD, Schonberger B, Jordan GH, Loening SA: Laparoscopic dismembered pyeloplasty: The method of choice in the presence of an enlarged renal pelvis and crossing vessels. Eur Urol 2002; 42(3):268-275.

Correspondencia autor: Dr. Alberto Parente Hernández Sección de Urología Pediátrica, Servicio Cirugía Pediátrica. Hospital Infantil Gregorio Marañón.

Maiquez, 9 - 28009 Madrid

Tel.: 915868000

E-mail autor: parente80@hotmail.com

Información artículo: Original - Pediatría

Trabajo recibido: agosto 2008

Trabajo aceptado: octubre 2008 